\title{
Tipología de las laderas del valle del Júcar (Albacete): Factores geomorfológicos e intervención antrópica
}

\author{
ANTONIO FERnÁNDEZ FERnÁNDEZ* Y VIRGINIA Rubio FERNÁNDEZ **
}

\begin{abstract}
RESUMEN
ABSTRACT

El río Júcar entre la población de

Valdeganga y el embalse de El Molinar, en el norte de la provincia de Albacete (fig. 1) se ha encajado en los estratos calizos de edad neógena de la comarca de La Manchuela. El valle presenta una morfología de hoz en el tramo alto y de cañón en el tramo más

bajo.

La dinámica de vertientes se ha manifestado de modo diferente a 10 largo del valle, al generar diversas formas y tipos de coluviones en sus distintos tramos, constituyendo las

laderas un importante elemento morfológico en el proceso de encajamiento del río.

Esta diversidad tipológica está relacionada con los factores que condicionan la dinámica de vertientes: geomorfológicos y antrópico. Los

The Jucar river, between the village of Valdeganga and El Molinar dam, to the north of the Albacete (fig. 1), has been embedded into limy strata of neogenic age in the La Manchuela region. The valley presents a gorge morphology in its upper section and a canyon morphology in its lower section.

Slope dynamics has shown in a different way along the valley, generating several forms and types of colluvial in its different sections, being the slopes an important morphological element in the river's embedding process.

This typological diversity is related to the factors which determine slope dynamics: geomorphological and antropic. The first ones show multiple interrelations with other variables of the physical medium, and are also

* UNED. Dpto. de Geografía

** Universidad Autónoma de Madrid.
\end{abstract}


primeros presentan múltiples interrelaciones con otras variables del medio físico e, igualmente, se encuentra muy mediatizados por la intervención antrópica. Ésta tiene una gran importancia ambiental e histórica por ser un elemento de elevada capacidad de alteración de la morfodinámica natural del valle y por la antigüedad del poblamiento en la comarca, detectado a partir de restos cerámicos en los coluviones del tramo alto del valle. affected by antropic intervention. This interference has great environmental and historial importance as it is an element highly able to alter the natural morphodynamics of the valley, and also because of the age of the region first settlements, detected from ceramic rests in colluvials located in the upper section of the valley.

\section{INTRODUCCIÓN}

El río Júcar entre la población de Valdeganga y el embalse de EI Molinar, en el norte de la provincia de Albacete (figura 1) se ha encajado en los estratos calizos de edad neógena de la comarca de La Manchuela. El valle presenta una morfología de hoz en el tramo alto y de cañón en el más bajo.

La dinámica de vertientes se ha manifestado de modo diferente a lo largo del valle, al generar diversas formas y tipos de coluviones en sus dis-

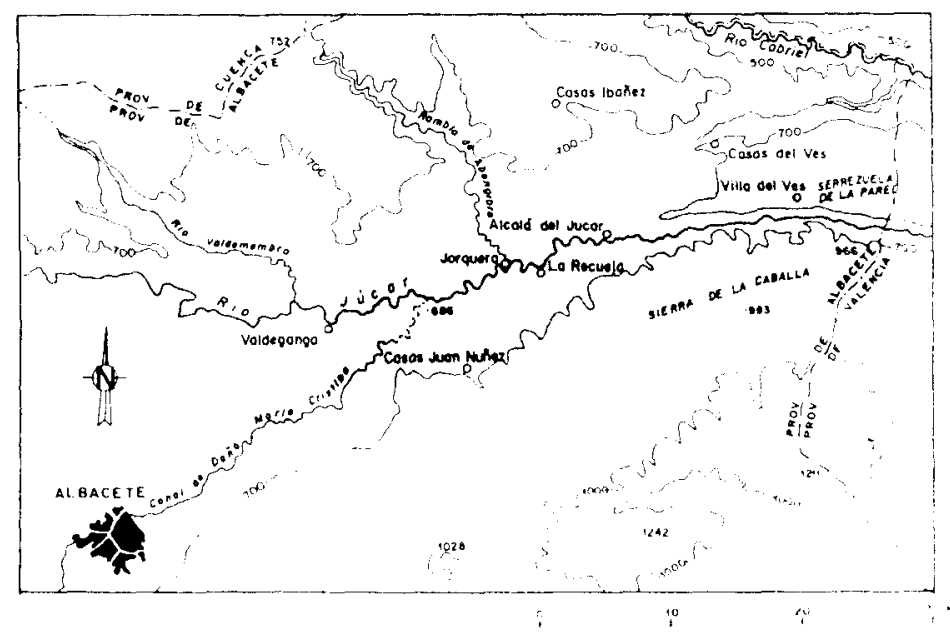

Figura 1. Localización del valle del Júcar (Albacete). 
tintos tramos, constituyendo las laderas un importante elemento morfológico en el proceso de encajamiento del río.

Esta diversidad tipológica está relacionada con los factores que condicionan la dinámica de vertientes: geomorfológicos y antrópicos. Los primeros presentan múltiples interrelaciones con otras variables del medio físico $e$, igualmente, se encuentran muy mediatizados por la intervención antrópica. Ésta tiene una gran importancia ambiental e histórica por ser un elemento de elevada capacidad de alteración de la morfodinámica natural del valle y por la antigüedad del poblamiento en la comarca, detectado a partir de restos cerámicos en los coluviones del tramo alto del valle.

El resultado final es una variada morfologia de vertientes en diferentes sectores del valle como puede observarse en la figura 2.

\section{DINÁMICA DE VERTIENTES Y FACTORES GEOMORFOLÓGICOS QUE LA CONDICIONAN}

Los factores geomorfológicos que condicionan la dinámica de vertientes se pueden agrupar en dos conjuntos: estructurales y dinámicos.

\section{a) Factores estructurales}

Están relacionados con la variedad litológica, la disposición tectónica y el grado de diaclasamiento de los estratos aflorantes.

El roquedo más representado en el valle es el correspondiente a un potente conjunto calizo de origen lacustre y edad pliocena, caracterizado por la alternancia de estratos micríticos y margosos, lo que favorece el desencadenamiento de procesos de erosión diferencial. Este conjunto ha recibido diversas denominaciones: Formación Río Júcar (Quesada, 1967 y Robles, 1974); Unidad Alcalá del Júcar (Ordoñez et al. 1975); Calizas Río Júcar (Bascones, 1978). Estas calizas afloran en la mayor parte del valle y originan múltiples modelos de vertiente, si bien, existe una morfología de hornacina que se reitera en los tramos del valle donde afloran estas calizas. Su presencia no es el producto de una evolución kárstica sino el resultado de la erosión diferencial, que al actuar con mayor intensidad en los estratos margosos, produce socavamientos y desprendimientos de paneles gravitatorios.

La ausencia de testigos tectónicos y la horizontalidad de los estratos del conjunto carbonático plioceno que predomina en los tramos superior y 

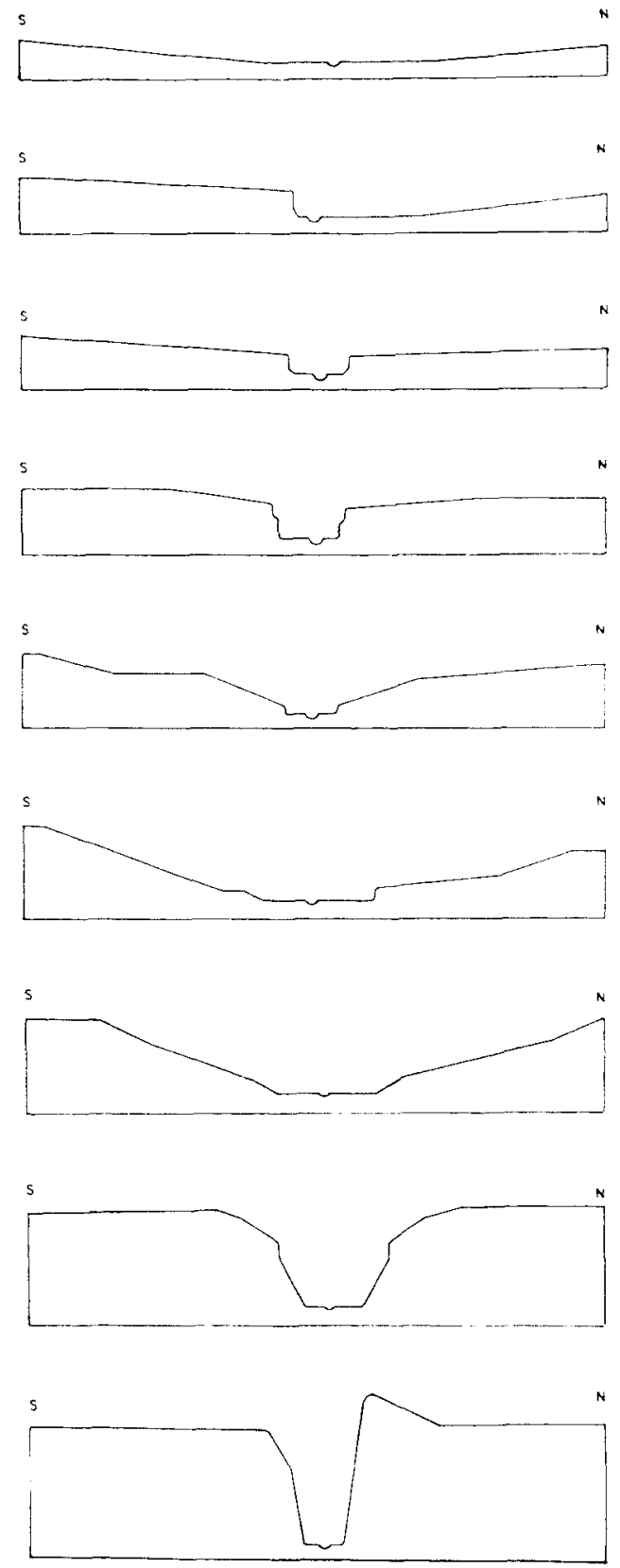

Figura 2. Morfología de las vertientes en diferentes tramos del valle. 
medio del valle contrasta con los buzamientos y densa red de fallas de las calizas y dolomías mesozoicas que afloran en su tramo inferior. En el primer caso la ausencia de tectonización queda sustituida por una red ortogonal de diaclasas verticales que se cruzan con los planos de estratificación horizontales. Ello provoca la liberación de pequeños clastos a partir de estos puntos de intersección. El resultado geomorfológico es el retroceso y erosión de la ladera, así como, la acumulación de derrubios que dan lugar a la formación de coluviones. En el tramo inferior del valle las laderas siguen una dinámica muy diferente debido a la fuerte tectonización del roquedo y a la propia naturaleza de éste.

\section{b) Factores dinámicos.}

Los procesos de vertientes y la acción fluvial establecen una interacción mutua, hasta el punto de condicionar sus propias evoluciones. Las laderas interfieren en la dinámica fluvial mediante la aportación de materiales detríticos que afectan a la capacidad de carga de la corriente. A su vez, la acción de las aguas del río provoca un socavamiento en la base de éstas lo cual desencadena readaptaciones de sus perfiles que pueden llegar a suponer la desaparición de los coluviones en las laderas.

El desnivel entre el fondo del valle y la llanura culminante se incrementa desde los escasos $10 \mathrm{~m}$ en Valdeganga (tramo superior), hasta algo más de $200 \mathrm{~m}$ en el tramo bajo en el embalse de El Molinar. Este hecho se manifiesta en un cambio sensible de la forma (figura 2) y las potencialidades gravitatorias de las vertientes a lo largo del valle.

Otro factor dinámico es el relacionado con la amplitud del valle y la posición del lecho fluvial. En los tramos donde el Júcar describe un curso meandriforme es visible la disminución del número de depósitos de ladera, e incluso, su ausencia. Este hecho es debido a la acción de socavamiento de la base de las vertientes efectuada por la corriente fluvial, especialmente en las márgenes cóncavas de los meandros. Por el contrario, en las convexas la incidencia de las acciones de socavación es muy reducida, lo que supone la acumulación de depósitos coluvionares que arrancan desde el sector culminante de la ladera y fijan su base en la plana aluvial. En los tramos rectilíneos la socavación lateral del río está en función de su caudal. Su variación está íntimamente relacionada con la intensidad pluviométrica y los desagües producidos por las centrales hidroeléctricas. Las laderas en estos tramos presentan una generalizada estabilidad que se puede ver interumpida por breves episodios de readaptación del perfil. 


\section{DINÁMICA DE VERTIENTES Y CONDICIONANTES DERIVADOS DE LA INTERVENCIÓN ANTRÓPICA}

La acción antrópica es el principal factor que condiciona, directa o indirectamente, la evolución morfodinámica de las laderas del valle. Su intervención indirecta supone la modificación de otros factores naturales que intervienen en los procesos de vertientes, como por ejemplo, la cubierta vegetal. Su acción directa acentúa los procesos erosivos, afectando al equilibrio de los taludes con construcciones de caminos, de viviendas semitroglodíticas o la desestabilización impuesta por el paso de ganado. La intervención humana en la comarca presenta datos históricos y arqueológicos desde tiempos muy remotos que demuestran la desforestación de la cobertera vegetal de las laderas; su evolución reciente y actual sólo se puede entender si se introduce la variable histórico-humana en su análisis.

\section{a) La deforestación de las laderas}

El desbroce de las laderas supone que éstas queden desprotegidas frente a los mecanismos de meteorización y erosión, dando lugar a un mayor aporte de materiales detríticos al cauce del río. El Mapa de Estados Erosivos de la Cuenca Hidrográfica del Júcar (ICONA) señala un potencial erosivo de 100-200 tm/ha/año. Esta cifra está en relación con el escaso recubrimiento vegetal y la irregularidad pluviométrica caracterizada por escasas pero intensas precipitaciones que activan cursos intermitentes de agua integrados en una densa red parafluvial.

El desbroce de las laderas presenta diferentes etapas (Fernández, A. 1998), la más antigua se corresponde con la Edad del Bronce como lo demuestra la existencia de restos cerámicos aparecidos en ciertos coluviones del valle. Es de suponer que estas primeras intervenciones no significaron grandes modificaciones en el funcionamiento morfodinámico del Júcar.

La segunda etapa coincide con el periodo histórico de dominación árabe y reconquista cristiana, las fuentes documentales son escasas, sin embargo, las Relaciones Topográficas de Felipe II son el principal documento histórico, a partir del cual, se puede reconstruir el paisaje vegetal. En ellas se menciona la existencia de grandes dehesas la mayor era la que estaba "...junto a un prado del río, junto a la ermita de San Lorenzo poblada de álamos muy altos...", en la actualidad esta dehesa ha desaparecido y sólo existe como tal, una alameda en las orillas del río. 
La tercera etapa en el desbroce de las laderas coincide con los años posteriores a la guerra civil y la intensificación del cultivo del esparto lo que produjo una fuerte antropización de las laderas de solana del valle; igualmente la presencia de una mayor cabaña ganadera caprina y ovina suponía un factor añadido a la movilización del material.

Las consecuencias del hecho anterior ha sido la existencia de unas laderas desprovistas de vegetación lo cual ha favorecido la instalación de procesos de abarrancamiento y la fácil evacuación del material al fondo del cauce coincidiendo con momentos de fuertes precipitaciones y arroyadas concentradas.

La dinámica de vertientes es activa y ocasionalmente produce desprendimientos de paneles gravitatorios que provocan daños materiales, como queda reflejado en un informe fechado en Albacete el 13 de junio de 1979 y realizado por el Ingeniero director de la sección de vías y obras de la Excma. Diputación de Albacete. En dicho informe se dan cuenta de desprendimientos acontecidos en Alcalá del Júcar en 1976 que afectaron a un serie de viviendas y corrales en el área cercana del camino de acceso de "La Asomada". Igualmente, se sugiere la necesidad de cuidar y vigilar las paredes del valle contra los accidentes y desprendimientos que pudieran presentarse. De este modo se informa que en la primavera de 1978, como consecuencia de los temporales se desprendió una roca "en el sitio denominado Parte Baja del Castillo y que cayó sobre una vivienda..." según consta en el Expediente $n . \cong 2$ Legajo 2.925 “Obras de contención en Alcalá del Júcar” (1980). Este hecho indica por un lado la asociación de desprendimientos y condiciones meteorológicas de intensas lluvias, pero sin duda, los efectos de estas precipitaciones se vería aminorado si la coberiera vegetal fuese densa y capaz de frenar los efectos de la escorrentía superficial.

Los daños no sólo han sido materiales sino que en algunos casos provocaron víctimas humanas como el desprendimiento de 1946 que causó trece muertos en la calle de los Desamparados en la localidad de Alcalá del Júcar.

\section{TIPOLOGÍA Y ANÁLISIS DE LAS LADERAS DEL VALLE.}

Los factores geomorfológico y antrópico han condicionado una variedad tipológica de laderas que atendiendo a su perfil se clasifican en cóncavas y rectilíneas.

Esta diferente tipología de vertientes cóncavas y rectilíneas está en relación al trazado fluvial. En las primeras (localizadas en el tramo meandri- 
forme del valle) su forma se corresponde a una lenta regularización caracterizada por la llegada de material coluvial de los tramos superiores y su deposición en la base de la ladera (Calvo, 1987). Las laderas que se apoyan en la margen convexa de un meandro presentan una fuerte estabilidad basal que favorece el mecanismo de acumulación en sus tramos inferiores. Por el contrario en las márgenes cóncavas los depósitos de ladera han desaparecido por la propia dinámica fluvial.

Las laderas rectilíneas se localizan en el tramo inferior de valle donde el río adopta un trazado menos sinuoso, la socavación lateral del río en ambas márgenes, supone mayores readaptaciones del perfil.

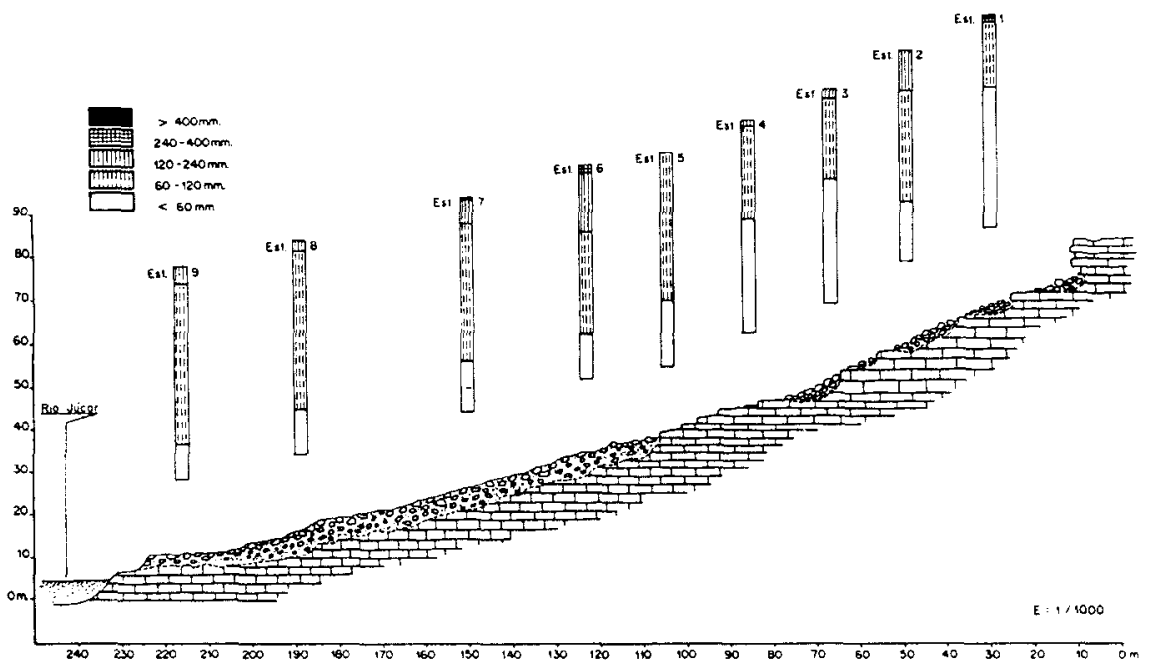

Figura 3. Ladera de la Recueja.

a) Laderas de pérfil cóncavo.

El modelo tomado como ejemplo es la ladera de La Recueja que toma su nombre de una aldea cercana. Sus depósitos detríticos se apoyan sobre los afloramientos de las calizas neógenas de Alcalá. (fig.3).

En lo referente a la cubierta vegetal destaca la completa ausencia de estrato arbóreo a lo largo de todo el perfil. Sólo aparece un matorral de esparto, boj y aliaga, que en el mejor de los casos se acerca al $40 \%$ de re- 
cubrimiento, en algunos de sus tramos. La parte basal de la ladera se encuentra desnuda de vegetación o con recubrimiento inferior al $10 \%$. Estos valores determinan que la vegetación no interfiera de forma significativa los procesos de dinámica de vertientes.

La ladera presenta una altura de casi $60 \mathrm{~m}$ y un perfil de $245 \mathrm{~m}$. En ella se pueden distinguir tres tramos en función del valor de su pendiente.

\section{a) Tramo superior.}

Se caracteriza por tener pendientes superiores a $20^{\circ}$ o valores muy cercanos a éste. Abarca una altura entre los 37-60 m. Sus coluviones están determinados por los aportes de clastos de pequeño tamaño procedentes de la meteorización de los escarpes de caliza que afloran entre ellos.

\section{b) Tramo medio}

Su pendiente media se sitúa entre $15-18^{\circ}$, y su cota altimetrica entre los 37 y $17 \mathrm{~m}$. En este tramo no aflora el substrato por lo que el tamaño de los clastos de los coluviones se relaciona más con la llegada y acumulación de material procedente de los niveles superiores.

\section{c) Tramo basal}

Su altura está comprendida entre los 0 y $17 \mathrm{~m}$. Su pendiente disminuye sensiblemente y se encuentra entre los $8^{\circ}$ de los segmentos inferiores y los $15^{\circ}$ que alcanza en los segmentos superiores. Estos valores suponen que adopte un perfil cóncavo y los clastos aumentan de tamaño (>200 $\mathrm{mm}$ ) respecto a los dos tramos anteriores.

En esta ladera es detectable el movimiento de material asociado a los procesos de transporte gravitatorio directo, lo que resulta favorecido por el escaso recubrimiento vegetal.

\section{b) Laderas de perfil rectilineo}

Las laderas que presentan este perfil se localizan en el tramo bajo del valle en el que el río describe un trazado rectilíneo y su fondo presenta 
una menor anchura. Dentro de esta tipologia se ha tomado como ejemplo la ladera denominada La Muela del Castillo (figura 4). Se sitúa aguas abajo de la aldea de Tolosa, sus tramos medio y superior se desarrollan sobre los estratos calizos neógenos, mientras que el basal lo hace sobre materiales conglomeráticos.

La altura de la ladera es de $84.5 \mathrm{~m}$ y su perfil mide $180 \mathrm{~m}$ con pendientes variables entre los 20 y casi $40^{\circ}$.

\section{a) Tramo superior}

Su límite inferior se sitúa a $59.1 \mathrm{~m}$, se caracteriza por una acusada pendiente con un valor medio de $32^{\circ}$ y máximo de $44^{\circ}$. En este tramo aflora el substrato que da lugar a pequeñas cornisas de menos de $2 \mathrm{~m}$ de altura y a partir de las cuales se libera material que será arrastrado ladera abajo.

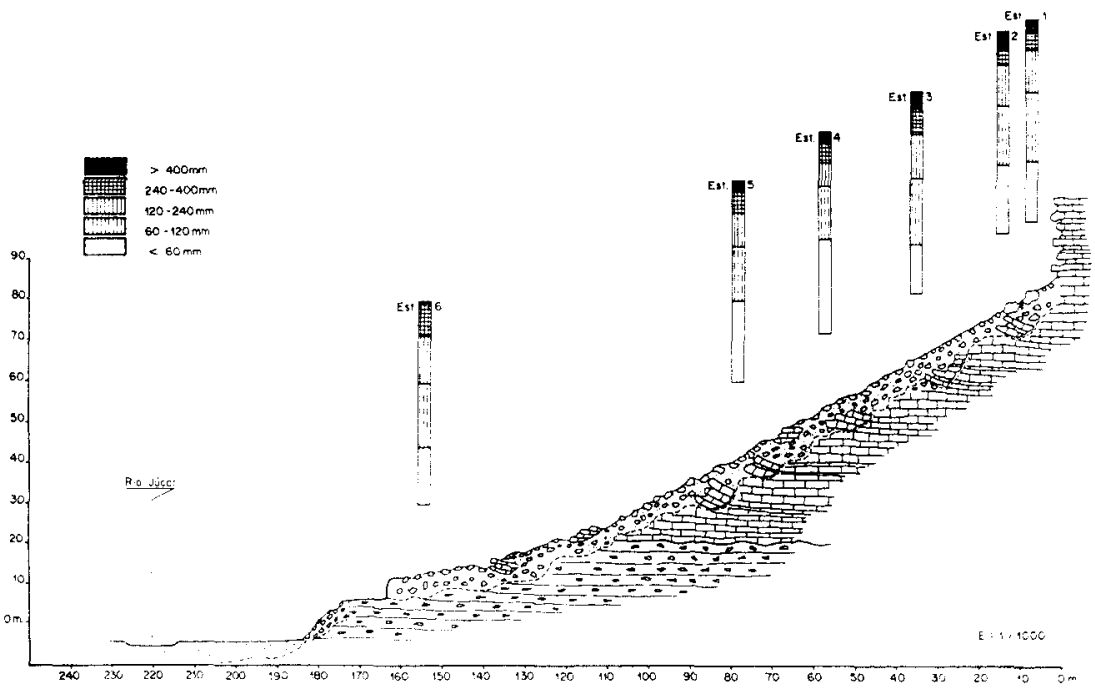

Figura 4. La Muela del Castillo.

El recubrimiento vegetal es relativamente elevado con presencia de estrato arbóreo que cubre un $50 \%$ de la superficie. El pinar que coloniza la ladera obedece a una antigua repoblación efectuada a mediados de los cuarenta. 
b) Tramos medio e inferior

Desde la cota $59.1 \mathrm{~m}$ hasta la base colocada en el fondo aluvial, la ladera adquiere una gran homogeneidad en su perfil al tener todos los valores de pendiente entre 20 y $29^{\circ}$, este hecho acentúa su carácter rectilíneo y la dificultad de delimitar un tramo basal por criterios de pendiente, aunque si por factores litológicos.

El perfil rectilíneo sólo se ve alterado por pequeñas rupturas de pendiente de carácter local originadas por la presencia de antiguos bloques gravitatorios de gran tamaño, cubiertos por derrubios más modernos. En ocasiones estos paneles gravitatorios afloran y producen las comentadas rupturas locales de pendiente (figura 4).

Esta ladera presenta indicios de inactividad y su análisis granulométrico muestra una heterogénea clasificación del material, resultado de, al menos, dos fases de formación.

Una más antigua en la que predominaba el transporte gravitatorio, consistente en el desprendimiento de grandes paquetes rocosos desde las cornisas del cañón que, más o menos fragmentados, descendieron hasta posiciones basales de la ladera (algunos de ellos se localizan a menos de $10 \mathrm{~m}$ de altura sobre el cauce actual).

La fase más moderna consistió en una acumulación de material integrado por clastos centimétricos que cubrieron los antiguos bloques gravitatorios y que en la actualidad conforman los coluviones que integran la ladera.

\section{CONCLUSIONES}

- En general las laderas del cañón del Júcar presentan una funcionalidad muy reducida. Los procesos gravitatorios libres son muy limitados, a pesar de estar, potencialmente favorecidos por una ausencia de fitoestabilización de las vertientes.

- El principal proceso de transporte es el asociado a la ocasionales e intensas precipitaciones de características mediterráneas. Éstas provocan una rápida escorrentía superficial, favorecida por la ausencia de vegetación, que moviliza el material desde las laderas hacia el río, lo que supone un progresivo desmantelamiento de los coluviones de los tramos superiores, y el consiguiente afloramiento del substrato al quedar libre de los derrubios que lo cubrían. 
- La intervención antrópica ha sido un elemento clave en la modificación del equilibrio ambiental y de la dinámica morfogenética de las laderas en el valle del Júcar. La desforestación de las vertientes ha facilitado los procesos de gravedad y de arroyada.

Igualmente, las múltiples construcciones semitroglodíticas que jalonan el valle favorecen la acción de desprendimientos locales que en llegan a producir daños humanos.

- La acción de la gravedad directa parece haber sido más competente en el pasado que en los momentos actuales en los que predominan los procesos de gravedad indirecta. Tanto en un momento como en otro, la preponderancia parece haber correspondido a los desprendimientos, más que a la "caída libre». Esto se encuentra en íntima relación con la estructura interna y la alta fragilidad presentada por las formaciones aflorantes, lo que favorece la disyunción y caída de grandes paneles rocosos que se fragmentan al tiempo que se desplazan por el perfil de la ladera.

\section{BIBLIOGRAFIA}

BRUCKNER, H. (1986) "Man's impact on the evolution of the physical environment in the mediterranean region in historical times". Geojournal n² 13, págs. 7-17.

FERNÁNDEZ FERNÁNDEZ, A. (1996): “Geomorfología del cañón del río Júcar en la comarca de La Manchuela (Albacete)". Tesis doctoral inédita. Universidad Complutense.

Fernández Fernández, A. (1998): Modificaciones antrópicas de la morfodinámica actual del Júcar. en "Medios carbonatado continentales" Edt. Universidad de Sevilla (en prensa).

Fernández, A., Garcia del CurA, M.A., González, J.A y Ordóñez, S.(1991): «El cañón del Júcar en La Manchuela". En libro: Guia de los Espacios Naturales de Castilla La Mancha. Servicio de Publicaciones de la Junta de Comunidades de Castilla La Mancha. 709 págs. 605-624. Toledo.

GonzÁlez MARTín, J.A. (1986): “Las laderas de los páramos alcarreños". En libro: Atlas de geomorfologia. Alianza Editorial, 365 págs.: 179-188. Madrid.

GonzÁlez MARTín, J.A. (1986): “Mapa geomorfológico 1/1.000.000 de la región de Castilla-La Mancha. En Atlas: Castilla La Mancha. Consejería Educación y Cultura. Junta de Comunidades de Castilla-La Mancha. págs.: 27, 72-73. Toledo.

González Amuchastegui, M.J. y González MARtin, J.A. (1990): "Derrubios crioclásticos y etapas frías en el área de Molina de Aragón" / Reunión Nacional de Geomorfología. págs.: 169-178. Teruel.

KOENIGUER,J.C. (1986): "Les changements climatiques au cours de l'historie des derniers millénaires dans les regions de la Méditerranée Occidentale" in LOPEZ VERA, F. (ed). Quaternary climate in Western Mediterranean. págs.: 594-563 Madrid.

LÓPEZ BERmúdez, F. (1992): “Deterioro ambiental en las tierras de regadio por plaguicidas y fertilizantes" en El medio rural español. Cultura, paisaje y naturaleza. Homenaje a A. Cabo Alonso, vol. I, págs.: 119-132.

MATEU Bel.LES, J.F. (1992): “Morfogénesis mediterránea en tiempos históricos: limitaciones de un debate geoarqueológico" en Servicio de investigación prehistórica. Diputación provincial de Valencia. $n^{\circ} 89$. Valencia.

Ordoñez, S.; Santos, J.A. y Garcia del Cura, M. ${ }^{\circledR}$ A. (1975): “Contribución al conocimiento de la evolución de la cuenca del río Júcar (España), durante el neógeno-cuaternario" en /I Congreso Iberoamericano. Geol. Econom. Sección II. págs.: 579-596.

Ordoñez, S; SAntos, J.A. y Garcia del CuRA, M.A. (1976) “Estudio sedimentológico de la cuenca del Júcar.l-Rocas carbonáticas". Estudios Geológicos. n² 32. págs.: 535-545. 
Tipología de las laderas del valle del Júcar (Albacete): Factores geomorfológicos...

Quesada, A.; Rev, R. y Escalante, G. (1967): "Reconocimiento geológico de la zona de Carcelén, (provincias de Albacete y Valencia). Boletín del I.G.M.E. n 78. págs.: 93-187.

Robles, F. (1970): "Estudio estratigráfico y paleontológico del Neógeno continental de la Cuenca del río Júcar". Tesis Doctoral. Universidad de Valencia.

ROBLES, F. (1974): "Bioestratigrafía continental del Neógeno superior y cuaternario inferior". Guía de excursiones del coloquio Internacional.

SanCHEZ ORTEGA, D. (1995): "Los LLanos de Albacete". Instituto de Estudios Albacetenses. Albacete.SANTOS, J.A. (1975): “Estudio petrológico del Terciario continental de la Cuenca del Júcar (Albacete)". Tesis de licenciatura. Universidad Complutense. Madrid.

SANTOS, J,.A. (1980): "Contribución al conocimiento litoestratigráfico y sedimentológico de la cuenca del Júcar (Albacete)". IX Congreso Nacional de Sedimentología. Resúmenes págs. 47-49.

THORNES, J.B. (1987): "The Paleo-ecology of Erosion" in WAGSTAFFJ.M (ed). en Landscape and culture. págs.: 37-55. Oxford. 